\title{
Edge State Magnetism of Single Layer Graphene Nanostructures
}

\author{
Somnath Bhowmick ${ }^{1 *}$ and Vijay B Shenoy, 2 团 \\ ${ }^{1}$ Materials Research Center, Indian Institute of Science, Bangalore 560 012, India \\ ${ }^{2}$ Centre for Condensed Matter Theory, Department of Physics, \\ Indian Institute of Science, Bangalore 560 012, India
}

(Dated: November 5, 2018)

\begin{abstract}
We study edge state magnetism in graphene nanostructures using a mean field theory of the Hubbard model. We investigate how the magnetism of the zigzag edges of graphene is affected by the presence of other types of terminating edges and defects. By a detailed study of both regular shapes, such as polygonal nanodots and nanoribbons, and irregular shapes, we conclude that the magnetism in zigzag edges is very robust. Our calculations show that the zigzag edges that are longer than three to four repeat units are always magnetic, irrespective of other edges, regular or irregular. We, therefore, clearly demonstrate that the edge irregularities and defects of the bounding edges of graphene nanostructures does not destroy the edge state magnetism.
\end{abstract}

\section{INTRODUCTION}

Graphene is a single layer of carbon atoms forming a densely packed honeycomb lattice. Novoselov et al. $\frac{1,2}{2}$ invented the top-down technique of isolating single layered graphene samples of a few microns in size by micromechanical cleavage of graphite. This advance has lead to a flurry of activity, both theoretical and experimental, towards understanding the physics of graphene $e^{\frac{3,4,5}{4}}$

A simple tight binding mode ${ }^{6} \underline{6}$ for electron hopping on the honeycomb lattice produces two bands which touch each other at two points in the Brillouin zone. The chemical potential of the undoped graphene lies exactly at the energy where the two bands touch, implying that graphene is a zero gap system with two distinct "Fermi points". What makes it even more interesting is the fact that the spectrum near these Fermi points resembles the Dirac spectrum of massless Fermions,,$\frac{7,8,9}{9}$ and the density of states depends linearly on the energy. Graphene shows several interesting magnetotransport properties, such as quantum Hall effect at room temperature $\stackrel{10}{=}$ unconventional quantum Hall effect,, 11 strongly suppressed weak localization magentoresistance 12 and quantum electrodynamics phenomena such as Klein paradox $\frac{13}{13}$ etc. Due to it's unusual electronic properties, graphene is a strong contender for future electronic applications. Examples

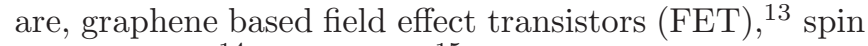
valve devices,$\stackrel{14}{\longleftarrow}$ gas sensors,$\frac{15}{\longmapsto}$ integrated ballistic carrier devices based on nanopatterned epitaxial graphene ${ }^{16}$ etc.

Interesting nanostructures can also be made from graphene. For example, a nanoribbon 17 is obtained by reducing the dimension of a graphene sheet along one direction to the nanometric size, and a nanodot by reducing both the dimensions to the nanometric sizes. Graphene nanostructures can be terminated by many different types of edges $\frac{17}{}$, for example, by "zigzag" or "armchair" edges (see FIG. 11). These edges can have a profound influence on the electronic structure, and can give rise to interesting new phenomena. For example, zigzag edges have localized electronic states with nearly flat dispersion, giving rise to the finite DOS at the chemical potential, as has been reported 17,18 from the the- oretical calculations. These have also been experimentally observed by scanning tunneling microscopy (STM) and spectroscopy $\underline{19,20}$. Indeed, the presence of the zigzag edges gives rise to unique physical and chemical properties as reported by Son et al. $\stackrel{21}{2}$ and Jiang et al. $\frac{22}{2}$ based on the first principle density functional calculations.

Focus of the present paper is on the edge state magnetism of graphene nanostructures. Based on first principle density functional calculations, it has been reported that the zigzag nanoribbons have an antiferromagnetic (AFM) ground state, with one edge spin up and the other spin down 23 , whereas the armchair edged nanoribbons do not show any such magnetic property. The investigation has further been extended to graphene nanodots of rectangular shape $e^{24}$ and regular hexagonal, as well as triangular nanodots 25 by first principle and mean field Hubbard model calculations; the results of these two approaches are found to be in agreement 25

All the reports so far deal with the nanodots terminated by consecutive zigzag edges. However, the widely used top-down technique $\mathrm{e}^{\underline{\underline{1}}}$ does not allow a careful control of the synthesis at the atomic level to produce graphene nanodots of regular shape, terminated, for example, entirely by zigzag edges. Furthermore, in spite of recent advances ${ }^{26}$, bottom-up techniques which are likely to give better control on the resulting structure are not yet widely practiced. The simplest defects to the "ideal" zigzag edged nanostructures are armchair terminations,$\underline{27}$ Despite the fact that there are several studies of defect induced magnetism in graphene, $\stackrel{27,28,29}{2}$ to the best of our knowledge, edge state magnetism of realistic graphene nanostructures, in particular those randomly terminated by the zigzag or armchair edges is yet to be examined in detail. In this work we address this issue and understand how the magnetism of zigzag edges "copes" with the presence of other edges and random terminations. Using an unrestricted Hartree-Fock mean-field theory of the Hubbard model on the graphene lattice, we study nanostructures with various different terminations with the aim of uncovering how the edge magnetism is affected. Three cases are investigated. First, we study nanodots by starting from a perfect hexagonal shape and systematically 


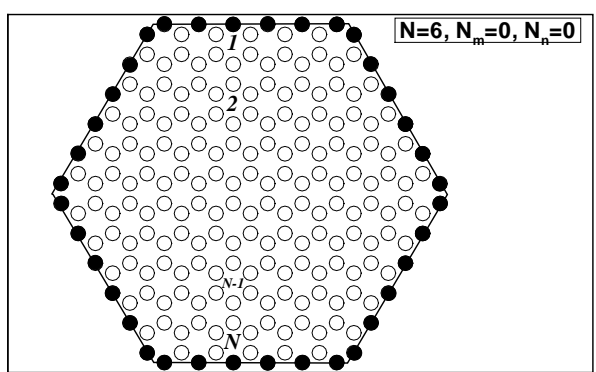

(a)

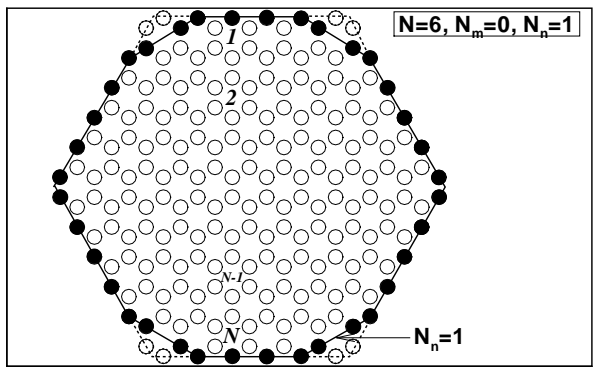

(c)

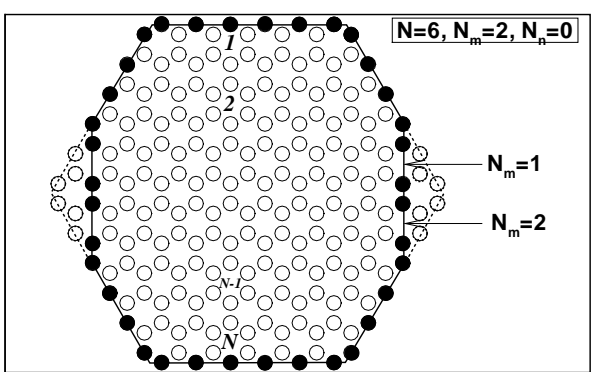

(b)

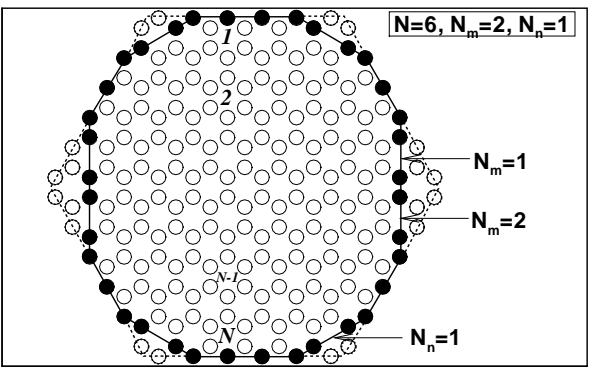

(d)

FIG. 1: (a) Hexagon: all the edges are of zigzag type. The size of the nanodot is denoted by $N$. We cut this polygon in different ways in order to produce various other polygons having both armchair and zigzag edges. Dark circles represent the edge sites in all the figures, and the dashed circles represent atoms that are removed. (b) Eight edged polygon: created by vertically cutting the hexagon resulting in two additional armchair edges per vertical side (quantified by $N_{m}$ ). The dotted portion in (b), (c) and (d) is the remaining part of the original full hexagon. (c) Ten edged polygon: obtained by cutting the hexagon along the slanted edge (no vertical cut) resulting in one armchair edge per slanted side (quantified by $N_{n}$ ) in the process. (d) Twelve edged polygon: created by simultaneously cutting along the vertical as well as the slanted edges, adding total four armchair edges of each type along the vertical and slanted directions respectively.

introducing other types of edges. Second, we investigate graphene nanoribbons terminated by the zigzag edges and systematically introduce "surface edge defects" and study the resulting magnetism. Third, we study "random" nanostructures by introducing completely irregular edges, while preserving some zigzag segments at the edges. The Hartree-Fock approach allows us to study large nanostructures with as many as 1000 carbon atoms; such a study within the first principles framework would require much bigger computational resources. However, we do not take into account effects like bond length variations and reconstructions at the edges, as well as effects of functional groups (e.g. oxidized edges) in the present calculation.

The remainder of the paper is organized as follows. In the next section we brief the mean field Hubbard model used in our study. This is followed by a section (Sec. III) where we present and discuss our results. In the concluding section (Sec. IV), we discuss the significance of our results particularly in the context of experimental observation of edge magnetism in graphene.

\section{MODEL DETAILS}

We study graphene within a tight binding model including the on-site Hubbard repulsion, i. e., within the framework of the Hubbard mode ${ }^{25,30,31}$, which has been used earlier to study surface magnetism ${ }^{32,33}$ The Hamiltonian is

$$
H=-t \sum_{\langle i j\rangle \sigma}\left(c_{i \sigma}^{\dagger} c_{j \sigma}+h . c\right)+U \sum_{i} n_{i \uparrow} n_{i \downarrow}
$$

where $c_{i \sigma}\left(c_{i \sigma}^{\dagger}\right)$ annihilates(creates) an electron of spin $\sigma$ at site $i, n_{i \sigma}=c_{i \sigma}^{\dagger} c_{i \sigma}$ is the number operator at site $i$, $t$ is the hopping amplitude, $U$ is the on-site Coulomb repulsion. The Hamiltonian has lattice translational and $\mathrm{SU}(2)$ spin rotation symmetries. Under the mean field approximation, we write the Hubbard Hamiltonian as

$H=-t \sum_{\langle i j\rangle \sigma}\left(c_{i \sigma}^{\dagger} c_{j \sigma}+h . c\right)+U \sum_{i}\left(n_{i \uparrow}\left\langle n_{i \downarrow}\right\rangle+n_{i \downarrow}\left\langle n_{i \uparrow}\right\rangle-\left\langle n_{i \downarrow}\right\rangle\left\langle n_{i \uparrow}\right\rangle\right)$

where $\left\langle n_{i \sigma}\right\rangle$ are the site occupations which are determined self consistently. We perform a completely unrestricted calculation keeping all site occupations $\left\langle n_{i \sigma}\right\rangle$ as unknowns. We focus on the undoped graphene, which had exactly one electron per site, i. e., we work with the half filled Hubbard model. From the self consistent ground state calculated numerically, we obtain the expectation value of $z$-component of the spin operator $S_{i}^{z}=\frac{1}{2} c_{i \alpha}^{\dagger} \sigma_{\alpha \alpha^{\prime}}^{z} c_{i \alpha^{\prime}}$ to study the resulting magnetic structure ( $\sigma^{z}$ is a Pauli matrix). 


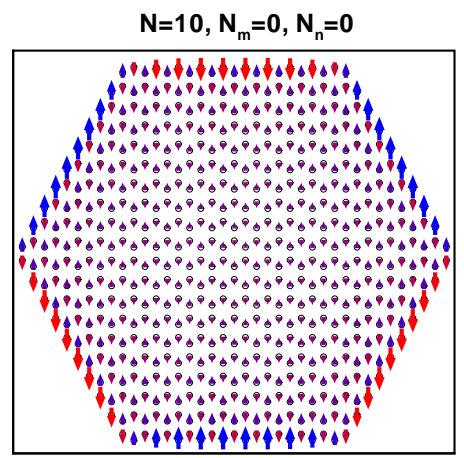

(a)

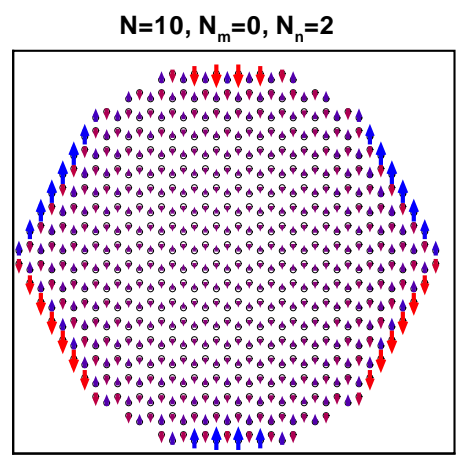

(c)

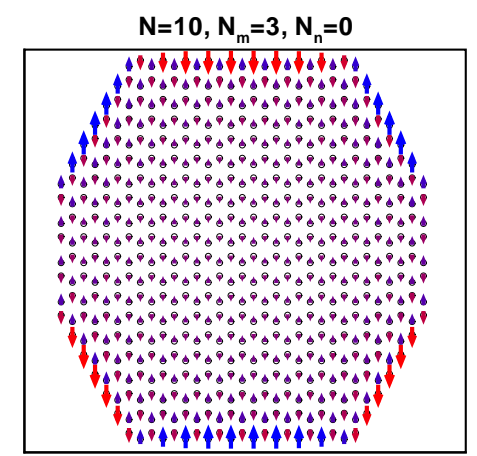

(b)

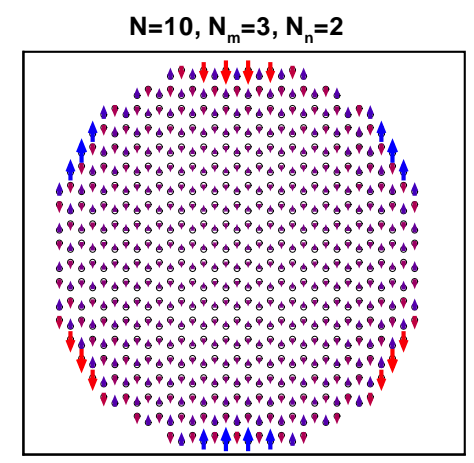

(d)

FIG. 2: (Online Color) Expectation value of $\left\langle S^{z}\right\rangle$ in four different graphene nanodots. The size of the arrows is proportional to the magnitude of magnetic moment. Note that there exist large magnetic moments at the zigzag edges compared to the sites lying inside or armchair edges.

We first discuss known results $\frac{34}{34}$ of the magnetic phases of the half filled Hubbard model on an infinite honeycomb lattice. The honeycomb lattice does not break any symmetry of the Hamiltonian until the onsite Coulomb repulsion attains a critical value $\underline{34}$ of $U_{c} / t \sim 2.23$. Above this value of the onsite Coulomb repulsion, the system breaks $\mathrm{SU}(2)$ spin rotation and lattice translational symmetries and develops an antiferromagnetic order (due to its bipartite nature). This is unlike the $2 \mathrm{D}$ square lattice (with the nearest neighbour hopping), where even an infinitesimally small $U$ will give rise to the antiferromagnetic spin density wave(SDW $)^{34}$ at half filling. This result of the honeycomb lattice may be understood starting from the magnetic susceptibility $\chi(q)$ obtained from the linear response theory, 35

$$
\chi(q)=(-1) \frac{1}{N} \sum_{k} \frac{f\left(\varepsilon_{k}-\mu\right)-f\left(\varepsilon_{k+q}-\mu\right)}{\varepsilon_{k}-\varepsilon_{k+q}}
$$

where $f(\varepsilon)$ is the Fermi function and $q$ is the wave vector of perturbing field, $\varepsilon_{k}$ is the electron dispersion, $\mu$ is the chemical potential. Now the half filled Hubbard model on a bipartite lattice with nearest neighbour hopping has particle-hole symmetry. Using eqn. (3), the bare mag- netic susceptibility (for the antiferromagnetic response) can be obtained as 35,36

$$
\chi_{0} \sim \int d \varepsilon \frac{\rho(\varepsilon)}{\varepsilon}
$$

where $\rho(\varepsilon)$ is the density of states(DOS). In case of a square lattice, $\rho(\varepsilon) \sim \log (\varepsilon)$ in the vicinity of chemical potential at zero doping and $\chi_{0}$ has a logarithmic divergence. As a consequence, the generalized Stoner criterion for anti ferromagnetic (AF) susceptibility,

$$
U \geq \frac{1}{\chi_{0}}
$$

is satisfied for even an infinitesimally small $U$ and $U_{c}=$ 0 for square lattice. However, in case of a honeycomb lattice, DOS depends linearly on the energy measured with respect to the chemical potential ${ }^{37}$,

$$
\rho(\varepsilon) \sim \varepsilon
$$

Hence the divergence of $\chi_{0}$ is absent (consult eqn. (4)), i.e., $\chi_{0}$ is a finite quantity. Antiferromagnetism, therefore, appears only for Coulomb repulsion greater than a 
critical value $U_{c}=1 / \chi_{0}=2.23 t$. Experimentally large graphene sheets do not show any magnetic ordering and hence the value of $U$ in graphene should be less than $U_{c}$ (this result, of course, does not include fluctuational corrections). We shall use a value of $U=2 t$ in our calculations, which at the mean field level will not produce any magnetic order in an infinite graphene sheet.

As noted in the introductory section, zigzag terminated nanoribbons (quasi 1D) possess special edge states which give rise to the flat bands and finite density of states at the Fermi level. 17,18,38 This leads to ferromagnetic spin orientations along a particular zigzag edge at infinitesimally small $U_{c}$ (from eqn. (4)). On the other hand, two opposite zigzag edges, terminating the nanoribbon at two opposite sides, are found to be aligned antiferromagnetically. The spin density (magnitude of the magnetic moment at a site) dies very quickly on moving into the "bulk", i.e., the centre of the nanoribbon - this is indeed edge state magnetism. Armchair edges do not support any magnetic structure for values of $U$ below $U_{c}$, in contrast to zigzag edges.

In the next section we shall investigate various graphene nanostructures terminated by zigzag and other edges with the aim of investigating how the presence of other edges affects the edge state magnetism.

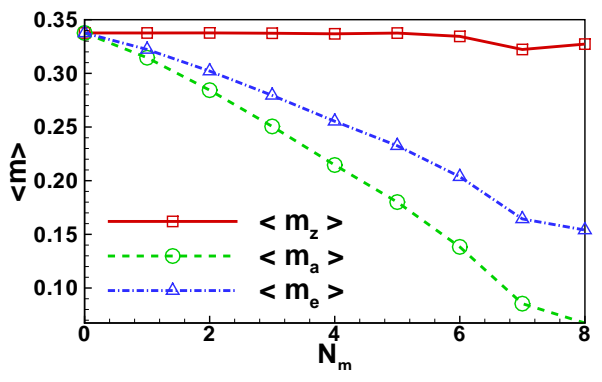

FIG. 3: (Online Color) Average magnetic moment per edge site for the eight edged polygons. $N_{m}$ is the number of armchair edges added along the vertical side (FIG. 1 (b)). $\left\langle m_{z}\right\rangle$ is the average magnitude of the magnetic moment per site along the purely zigzag (horizontal sides in FIG. 1(b) and FIG. 2(b)) edges. $\left\langle m_{a}\right\rangle$ is the average magnitude of the magnetic moment per site along the non-horizontal edges. $\left\langle m_{e}\right\rangle$ is the magnitude of the magnetic moment per site averaged over all the edge sites (zigzags as well as armchairs). As we increase $N_{m}$, we add armchairs at the non-horizontal sides and hence $\left\langle m_{a}\right\rangle$, as well as $\left\langle m_{e}\right\rangle$, decreases. Moreover, in the process of increasing $N_{m}$, we do not disturb the purely zigzag horizontal edges and $\left\langle m_{z}\right\rangle$ remains almost unaffected.

\section{GRAPHENE NANOSTRUCTURES: EDGE STATE MAGNETISM}

We study edge state magnetism in three types of nanostructures. Regular nanodots with zigzag and armchair edges, nanoribbons with "defected" zigzag edges, and random nanostructures with some zigzag segments.

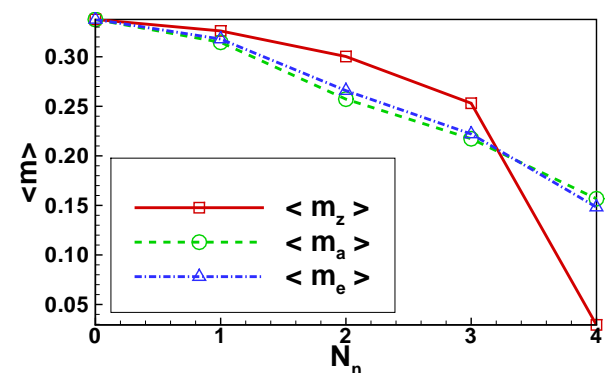

FIG. 4: (Color online) Average magnetic moment per edge site for the ten edged polygons. $N_{n}$ is the number of armchair edges added along the slanted sides of the hexagonal polygon (FIG. 1(c)). Consult FIG. 3 for the definition of $\left\langle m_{z}\right\rangle,\left\langle m_{a}\right\rangle$ and $\left\langle m_{e}\right\rangle$. Overall edge magnetization diminishes with the increase of number of armchairs. In particular, as we increase $N_{n}$ the horizontal zigzag segments are reduced in length and the most notable change occurs for $N_{n}$ greater than three, where the magnetization of the horizontal edges $\left(\left\langle m_{z}\right\rangle\right)$ essentially vanishes. Note that for $N_{n}=3$ there are four repeat units in the horizontal zigzag segment and for $N_{n}=4$ the zigzag segments are two repeat units long.

Before discussing each nanostructure in detail, we comment on the nature of the expected ground state. Total spin $S_{z}$ of the ground state of regular nanodots is found $^{25}$ to be zero and this result is consistent with the predictions of Lieb's theorem ${ }^{39}$ This arises from the fact that the earlier calculations with regular nanodots will have equal number of atoms of the "A-sublattice" and the "B-sublattice". On the other hand for irregular nanostructures, $N_{A}$ need not be equal to $N_{B}$, for example see FIG. 7(c) and (d) and total $S_{z}$ is expected (and found) to be nonzero in such cases.

\section{A. Nanodots}

Starting from a perfect hexagonal nanodot, 25 which is entirely enclosed by zigzag edges (FIG. 1)(a)), we can make various different polygons, that are terminated by both zigzags and armchair edges (see FIG. 17(b), (c),(d)). In this process we retain zigzag edges on the horizontal sides of the resulting polygon. Nomenclature of size etc. is explained in FIG. 1. The sizes of the horizontal zigzag segments can by controlled by varying the lengths of the armchair segments $N_{m}$ and $N_{n}$.

The magnetic structure obtained from the mean field analysis of the Hubbard model is shown in FIG. 2 . We observe that the surface sites, in particular those along the zigzag edges but not along the armchair edges, have large expectation values of the $\left\langle S_{z}\right\rangle$ operator. Moreover, the magnetic moment decreases sharply as we move towards the centre of the nanodot and at the "bulk" sites, it is in general at least one to two order of magnitude smaller in comparison to that at the zigzag edge sites. In all cases we find that the moment is along the "up direction" on sites of one sub-lattice and "down direc- 


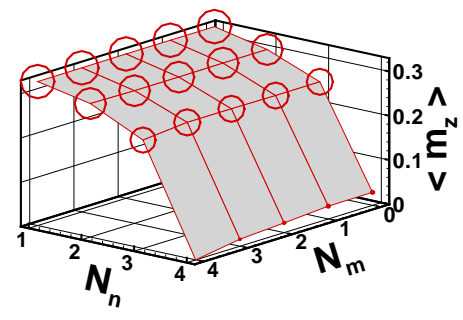

(a)

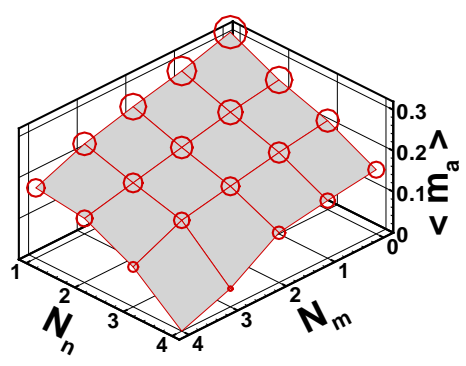

(b)

FIG. 5: (Color online) Average magnetic moment per edge site for the twelve edged nanodots (FIG. 11(d)). See FIG. 3 for the definition of $\left\langle m_{z}\right\rangle$ and $\left\langle m_{a}\right\rangle$. The size of the symbols is proportional to the magnitude of the average magnetic moment. (a) The key finding is that the magnetic moment along the horizontal zigzag edges is affected by only their length and not by the presence of other edges. Increase of $N_{m}$ does not affect the length of the horizontal zigzag edges, while increase of $N_{n}$ decreases the length of the horizontal edges. (b) $\left\langle m_{a}\right\rangle$, average magnitude of magnetic moment along the nonhorizontal sides decreases as we increase $N_{m}$ and $N_{n}$, thereby adding more armchairs to the edges.

tion" on the sites belonging to the other sub-lattice. We shall henceforth focus on the magnitude of the magnetic moment, keeping in mind this observation.

We now present a quantitative analysis of the how the magnetism at the horizontal zigzag edges is affected by the introduction of other segments. FIG. 3, FIG. 4 and FIG. 5 show the plots of the magnitude of the magnetic moments per site for the three types of regular polygons constructed from a hexagonal nanodot discussed in FIG. 1. The average magnitude of the magnetic moment per site along the zigzag segments is denoted by $\left\langle m_{z}\right\rangle$, while $\left\langle m_{a}\right\rangle$ denotes the average of the magnitude of the magnetic moment obtained on non-horizontal edges (the non-horizontal edges contain a mixture of both zigzag and armchair edges depending of $N_{m}$ and $N_{n}$ ). The average magnitude of the magnetic moment over all the edge sites is denoted by $\left\langle m_{e}\right\rangle$. FIG. 3 shows the variation of various average magnetic moments for the eight edged nanostructure of shown in FIG. 1(b). We find that the magnetization of the horizontal zigzag edges is unaffected by the presence of additional non-horizontal edges. The average magnitude of the magnetization $\left\langle m_{e}\right\rangle$ along all edges falls due to the presence of additional armchair segments. In FIG. 4 we show the results of average magnetization for the ten sided polygons. In this case the addition of non-horizontal armchair edges results (i. e., increase of $N_{n}$ ) in a reduction in the length of the horizontal zigzag segments. In this case we see that the magnetization of the zigzag segments falls (and so does the average magnetization) and for $N_{n}>3$, it essentially vanishes. The case of $N_{n}=3$ corresponds to having horizontal zigzag segments whose length is four repeat units. We turn now to the twelve sided polygons shown in FIG. 1(d) where we introduce two types nonhorizontal armchair segments denoted by $N_{m}$ and $N_{n}$. As in the case of the eight sided polygon the length of the horizontal zigzag segment is unaffected by increase of $N_{m}$ while the length of the horizontal zigzag segments is reduced up on increase of $N_{n}$. We see that the magnetization of the horizontal edges is unaffected by increase of $N_{m}$, but strongly affected by increase of $N_{n}$ which reduces the length of the horizontal zigzag edges. The upshot of our calculation is that the zigzag edges retain a significant amount of magnetization even in the presence of other edges as long as the length of the zigzag segments is greater than three to four repeat units. The number of repeat units of the zigzag edges that preserve the magnetization depends on the value of $U$. For example, our calculations show that for $U=1.2 t$, the number of repeat units must be five to six for retaining the magnetization.

\section{B. Nanoribbons}

The nanodots discussed in the last section had "undisturbed" zigzag edges. It is interesting to investigate if "defects" present in a zigzag edge can destroy the magnetism. We investigate this issue by considering nanoribbons which have a large aspect ratio. We start with nanoribbons enclosed solely by the zigzag edges, and introduce "armchair defects" along the long edges of the nanoribbon (see FIG. 6). The number of armchair defects introduced is denoted by $N_{a}$ while the spacing between these defects is denoted by $N_{d}$. Therefore if the value of $N_{d}$ is smaller, the number of armchairs $N_{a}$ is higher and vice versa. Here we define $\left\langle m_{z}\right\rangle$ as the average magnitude of the magnetic moment along the short edges, $\left\langle m_{a}\right\rangle$ as the average magnetic moment along the long edges possibly containing armchair defects. FIG. 6(d) shows a plots of these quantities. We observe from FIG. 6(d) that the moment along the short sides (which are defect free) is essentially unaffected by the number of defects on the long 


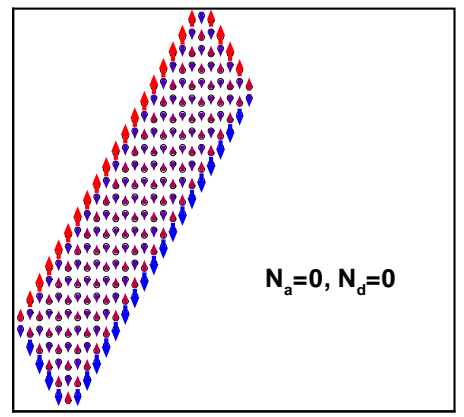

(a)

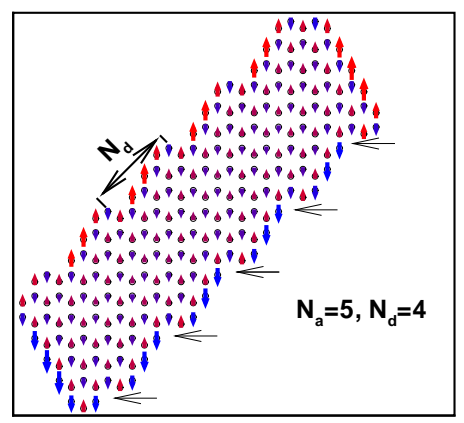

(c)

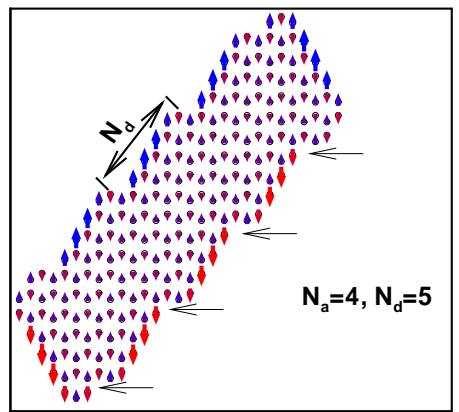

(b)

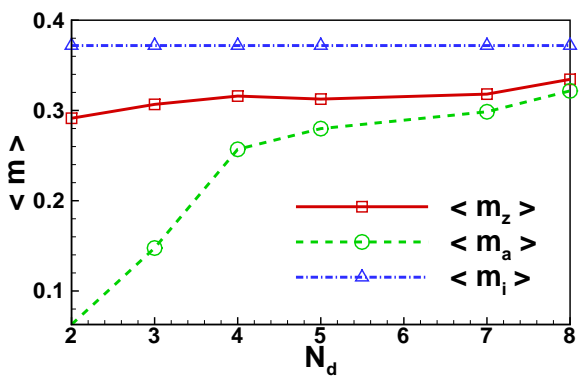

(d)

FIG. 6: (Color online) Magnetic structure of graphene nanoribbons. Nanoribbon in (a) is enclosed by the zigzag edges. The long zigzag edges of the nanoribbons (b) and (c) is broken by intermittent armchair "defects". As we decrease $N_{d}$, the interval between the armchair "defects" (i.e., increase $N_{a}$, the total number of armchair defects along the long side), the magnitude of the average localized magnetic moment along the long side (represented by $\left\langle m_{a}\right\rangle$ ) decreases. But $\left\langle m_{z}\right\rangle$, average localized moment along the short side remains unaffected. We denote average moment per edge site of the ideal zigzag structure (illustrated in (a)) by $\left\langle m_{i}\right\rangle$ in (d). Note the sharp drop of $\left\langle m_{a}\right\rangle$ below $N_{d}=4$, which is the limit of three zigzag repeat units.

side. However $\left\langle m_{a}\right\rangle$ falls gradually with the decrease of $N_{d}$ (increase of the number of the armchair defects) until $N_{d}$ reaches about 4 , where there is a dramatic fall in $\left\langle m_{a}\right\rangle$. Interestingly, this value of $N_{d}$ corresponds the situation where the contiguous zigzag segments in the "defected zigzag edge" have about three to four repeat units. We therefore find a result that is fully consistent with that found in the case of regular polygon nanodots; as long as there are three to four repeat units of zigzag edge present, the ferromagnetism on the edge is robust.

\section{C. "Irregular" Nanostructures}

We shall now investigate several "irregular" nanodots created by "cutting out" patches out of a graphene sheet. Such a random cutout can produce edge atoms which have two nearest neighbours, or a single nearest neighbour. In the latter case the structure thus obtained may not be an equilibrium structure; we ignore this compli- cation for the present study and focus on the nature of edge magnetism that arises in irregular structures. A study of FIG. 7 shows that the edge magnetism is very robust even in irregular structures; we find again that as long as there are three to four repeat units of a zigzag edge present, the edge magnetism is significant. We find an additional interesting feature. The sites with a single nearest neighbour (such as those that are indicated by a solid arrow in FIG. 7) have very large magnetic moments, larger than even the zigzag edges. As noted above, these are the "high energy edges" and are not likely to be supported by thermodynamics.

\section{CONCLUSION}

As noted in the introduction there have been a large number of theoretical efforts investigating edge state magnetism $23,24,25,40,41$, in regular nanostructures terminated by zigzag edges. However, we are not aware 


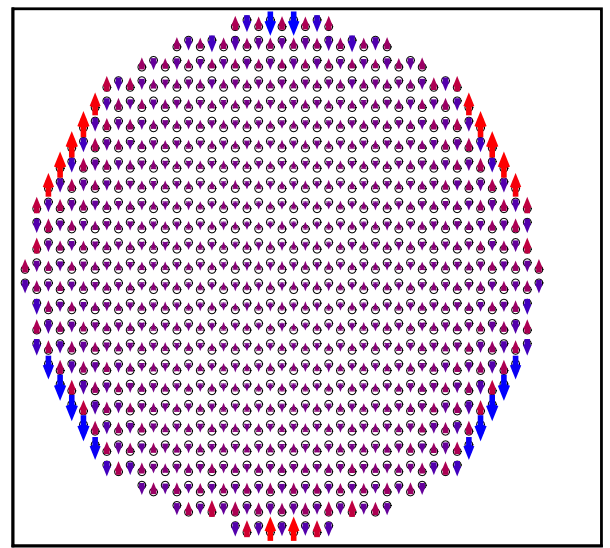

(a)

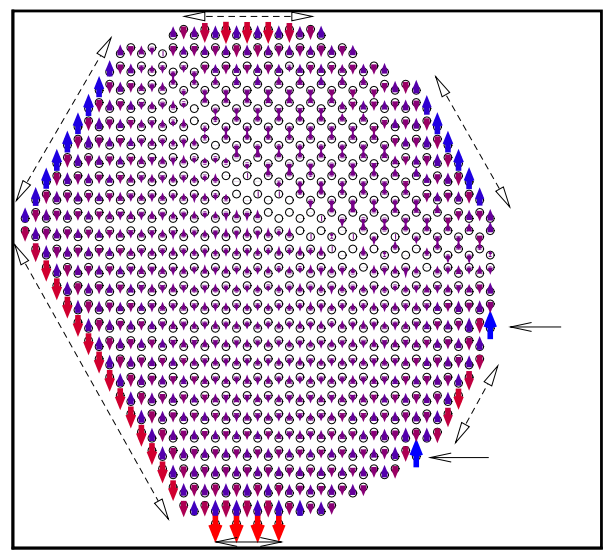

(c)

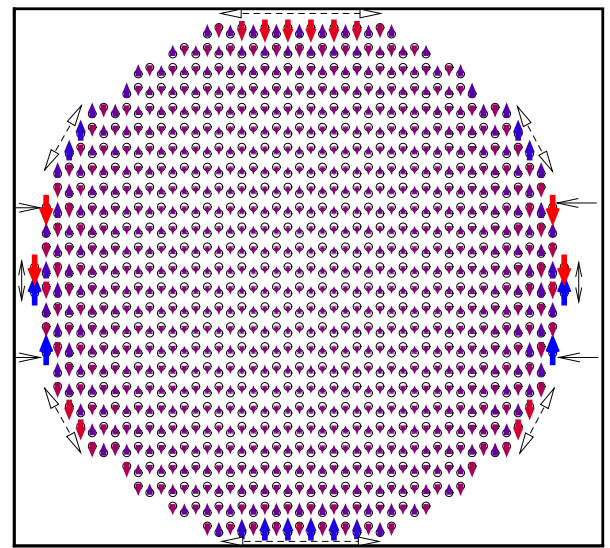

(b)

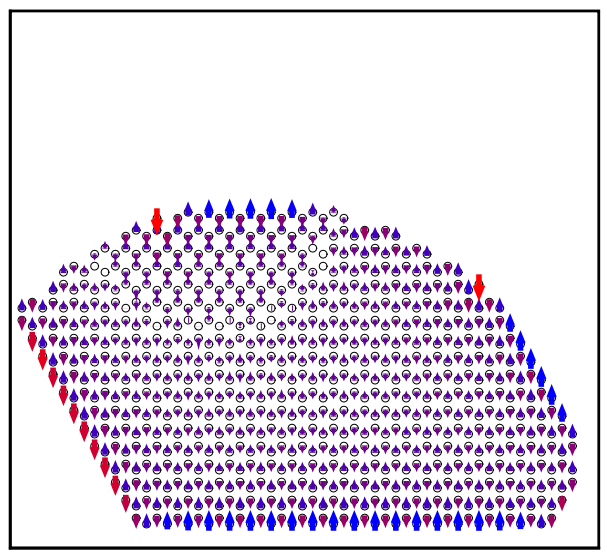

(d)

FIG. 7: (Color online) Magnetic structure of irregular graphene nanodots. (a),(b) "Circular cut-out" nanodots. There are two different type of sites where the magnetic moment is high. First, the usual zigzag edges, lying along the horizontal side and marked by dashed arrow. Second, a set of sites, marked by solid arrows, have only one nearest neighbor, as opposed to two in case of zigzags and armchair edges. The second set of sites have even higher magnetic moment than compared to the zigzag sites. (c),(d) - nanodots with both regular edges and a "circular boundary".

of any direct experimental observation of this phenomena for graphene nanoribbons or nanodots. There are a few experimental reports of magnetism in activated carbon fibers ${ }^{42.43}$, composed of a disordered network of nanographite, which remains to be the best proof of the theoretical prediction so far. In this paper we have enquired if "irregularity" of the experimental graphene nanostructures could be behind the lack of direct experimental corroboration. We have investigated how the edge state magnetism of the zigzag edges is affected by the presence of other edges, "defects" and random terminations. We find that the edge state magnetism is very robust to these "mutilations" of the nanostructures - for a value of $U=2 t$, as long as there are three to four repeat units of a zigzag edge, the edge state magnetism is preserved. For smaller values of on site Coulomb repulsion, more number of repeat units of a zigzag edge is required for sustaining edge state magnetism (e.g., for $U=1.2 t$, the critical number of repeat units is five to six). In addition, we note that certain "high energy" edges (ones where the edge atoms have only one nearest neighbour) can have very large moments compared to even the zigzag edges. Thus our study demonstrates that the shape irregularity is unlikely to destroy the edge 
state magnetism. However, it is clear that atomic resolution magnetic force microscopy may have to be employed for observation of edge state magnetism in short zigzag segment of graphene 44 On the theoretical front, the present approach is based on the mean field theory which, of course, neglects quantum fluctuation effects. These may be expected to be important in an "effectively 1D" system like the zigzag edge of graphene and have to be investigated further.

Note added: After completion of the manuscript, we became aware of a similar work by H. Kumazaki et al $\stackrel{45}{ }$
We thank Kumazaki et al. for bringing this to our notice.

\section{ACKNOWLEDGMENT}

The authors thank G. Baskaran, R. Shankar and U. Waghmare for discussions. The authors are particularly grateful to A. Ghosh for discussions, suggestions and comments. VBS thanks DST for generous support for this work through a Ramanujan grant.
* bsomnath@mrc.iisc.ernet.in

† shenoy@physics.iisc.ernet.in

1 K. S. Novoselov, A. K. Geim, S. V. Morozov, D. Jiang, Y. Zhang, S. V. Dubonos, I. V. Grigorieva, and A. A. Firsov, Science 306, 666 (2004).

${ }^{2}$ K. S. Novoselov, D. Jiang, F. Schedin, T. J. Booth, V. V. Khotkevich, S. V. Morozov, and A. K. Geim, Proc. Natl. Acad. Sci. USA 102, 10451 (2005).

3 A. K. Geim and K. S. Novoselov, Nat. Mater. 6, 183 (2007).

${ }^{4}$ M. I. Katsnelson, Materials Today 10, 20 (2007).

5 A. H. C. Neto, F. Guinea, N. M. R. Peres, K. S. Novoselov, and A. K. Geim, e-print arXiv:cond-mat/0709.1163v2 (2008).

6 P. R. Wallace, Phys. Rev. 71, 622 (1947).

7 G. W. Semenoff, Phys. Rev. Lett. 53, 2449 (1984).

8 F. D. M. Haldane, Phys. Rev. Lett. 61, 2015 (1988).

9 K. S. Novoselov, A. K. Geim, S. V. Morozov, D. Jiang, M. I. Katsnelson, I. V. Grigorieva, S. V. Dubonos, and A. A. Firsov, Nature (London) 438, 197 (2005).

10 K. S. Novoselov, Z. Jiang, Y. Zhang, S. V. Morozov, H. L. Stormer, U. Zeitler, J. C. Maan, G. S. Boebinger, P. Kim, and A. K. Geim, Science 315, 1379 (2007).

11 K. S. Novoselov, E. McCann, S. V. Morozov, V. I. Fal'ko, M. I. Katsnelson, U. Zeitler, D. Jiang, F. Schedin, and A. K. Geim, Nat. Phys. 2, 177 (2006).

12 S. V. Morozov, K. S. Novoselov, M. I. Katsnelson, F. Schedin, L. A. Ponomarenko, D. Jiang, and A. K. Geim, Phys. Rev. Lett. 97, 016801 (2006).

13 M. I. Katsnelson, K. S. Novoselov, and A. K. Geim, Nat. Phys. 2, 620 (2006).

14 E. W. Hill, A. K. Geim, K. Novoselov, F. Schedin, and P. Blake, Magnetics, IEEE Transactions on Magnetics 42, 2694 (2006).

15 F. Schedin, A. K. Geim, S. V. Morozov, E. W. Hill, P. Blake, M. I. Katsnelson, and K. S. Novoselov, Nat. Mater. 6, 652 (2007).

16 C. Berger, Z. Song, T. Li, X. Li, A. Ogbazghi, R. Feng, Z. Dai, A. Marchenkov, E. Conrad, P. First, et al., J. Phys. Chem. B 108, 19912 (2004).

17 K. Nakada, M. Fujita, G. Dresselhaus, and M. S. Dresselhaus, Phys. Rev. B 54, 17954 (1996).

18 K. Kobayashi, Phys. Rev. B 48, 1757 (1993).

19 Y. Kobayashi, K. Fukui, T. Enoki, K. Kusakabe, and Y. Kaburagi, Phys. Rev. B 71, 193406 (2005).

20 Y. Niimi, T. Matsui, H. Kambara, K. Tagami, M. Tsukada, and H. Fukuyama, Phys. Rev. B 73, 085421 (2006).

21 Y. W. Son, M. L. Cohen, and S. G. Louie, Nature (London)
444, 347 (2006)

22 D. en Jiang, B. G. Sumpter, and S. Dai, J. Chem. Phys. 126, 134701 (2007).

23 S. Okada and A. Oshiyama, Phys. Rev. Lett. 87, 146803 (2001).

24 D. en Jiang, B. G. Sumpter, and S. Dai, J. Chem. Phys. 127, 124703 (2007).

25 J. Fernandez-Rossier and J. J. Palacios, Phys. Rev. Lett. 99, 177204 (2007).

26 J. Wu, W. Pisula, and K. Mullen, Chem. Rev. 107, 718 (2007).

27 O. V. Yazyev and M. I. Katsnelson, Phys. Rev. Lett. 100, 047209 (2008).

28 O. V. Yazyev, e-print arXiv:cond-mat/0802.1735v2 (2008).

29 O. V. Yazyev and L. Helm, Phys. Rev. B 75, 125408 (2007).

30 G. Baskaran, Phys. Rev. B 65, 212505 (2002).

31 A. M. Black-Schaffer and S. Doniach, Phys. Rev. B 75, 134512 (2007).

32 M. Potthoff and W. Nolting, Phys. Rev. B 52, 15341 (1995).

33 M. Potthoff and W. Nolting, Phys. Rev. B 55, 2741 (1997).

34 N. Furukawa, arXiv.org:cond-mat/0103184v3 (2001).

35 M. Rasetti, The Hubbard Model Recent Results (World Scientific, 1991).

36 G. D. Mahan, Many-particle Physics (Kluwer Academic/Plenum Publishers, New York, 2000).

37 N. M. R. Peres, M. A. N. Araujo, and D. Bozi, Phys. Rev. B 70, 195122 (2004).

38 E. V. Castro, N. M. R. Peres, J. M. B. L. dos Santos, A. H. C. Neto, and F. Guinea, Phys. Rev. Lett. 100, 026802 (2008).

39 E. H. Lieb, Phys. Rev. Lett. 62, 1201 (1989).

40 E. V. Castro, N. M. R. Peres, and J. M. B. L. dos Santos, e-print arXiv:cond-mat/0801.2788v1 (2008).

41 K. Wakabayashi and K. Harigaya, J. Phys. Soc. Jpn. 72, 998 (2003).

42 Y. Shibayama, H. Sato, T. Enoki, and M. Endo, Phys. Rev. Lett. 84, 1744 (2000).

43 T. Enoki and Y. Kobayashi, J. Mater. Chem. 15, 3999 (2005).

44 U. Kaiser, A. Schwarz, and R. Wiesendanger, Nature 446, 522 (2007).

${ }^{45}$ H. Kumazaki and D. S. Hirashima, J. Phys. Soc. Jpn. 77, 044705 (2008). 